\title{
Family Medicine in Ethiopia: Lessons from a Global Collaboration
}

\author{
Ann Evensen, MD, FAAFP, Dawit Wondimagegn, MD, Daniel Zemenfes Ashebir, MD, \\ Katherine Rouleau, MDCM, CCFP, MHsc, Cynthia Haq, MD, \\ Abbas Ghavam-Rassoul, MD, Praseedha Janakiram, MD, CCFP, \\ Elizabeth Kvach, MD, MA, Heidi Busse, PhD, MPH, James Conniff, MD, MPH, \\ and Brian Cornelson, MD, CCFP
}

Background: Building the capacity of local health systems to provide high-quality, self-sustaining medical education and health care is the central purpose for many global health partnerships (GHPs). Since 2001, our global partner consortium collaborated to establish Family Medicine in Ethiopia; the first Ethiopian family physicians graduated in February 2016.

Methods: The authors, representing the primary Ethiopian, Canadian, and American partners in the GHP, identified obstacles, accomplishments, opportunities, errors, and observations from the years preceding residency launch and the first 3 years of the residency.

Results: Common themes were identified through personal reflection and presented as lessons to guide future GHPs.

LESSON 1: Promote Family Medicine as a distinct specialty.

LESSON 2: Avoid gaps, conflict, and redundancy in partner priorities and activities.

LESSON 3: Building relationships takes time and shared experiences.

LESSON 4: Communicate frequently to create opportunities for success.

LESSON 5: Engage local leaders to build sustainable, long-lasting programs from the beginning of the partnership.

Conclusions: GHPs can benefit individual participants, their organizations, and their communities served.

Engaging with numerous partners may also result in challenges—conflicting expectations, misinterpretations, and duplication or gaps in efforts. The lessons discussed in this article may be used to inform GHP planning and interactions to maximize benefits and minimize mishaps. (J Am Board Fam Med 2017;30:670-677.)

Keywords: Ethiopia, Family Physicians, Global Health, Internship and Residency, Program Evaluation

Introduction

\section{Global Health Partnerships}

The United Nations Sustainable Development Goals emphasize health as a prerequisite for equitable and sustainable global development. ${ }^{1}$

This article was externally peer reviewed.

Submitted 28 February 2017; revised 12 May 2017; accepted 31 May 2017.

From the Department of Family Medicine and Community Health (AE, CH, JC), Department of Surgery (HB), University of Wisconsin School of Medicine and Public Health, Madison, WI; Department of Psychiatry (DW), Department of Family Medicine (DZA), Addis Ababa University, Addis Ababa, Ethiopia; St-Michael's Hospital, Department of Family and Community Medicine, University of Toronto, Canada (KR, AGR, PJ, BC); Department of Family Medicine, University of Colorado-Denver, Denver,
Recognizing the need to address health disparities and strengthen health care systems specifically in low- and middle-income countries, agencies and

CO (EK); Department of Family Medicine, University of Calgary, Calgary, Canada (BC).

Funding: No financial support was provided for writing and development of this manuscript but the following institutions provided direct or in-kind support of the Addis Ababa University Family Medicine Program: University of Toronto, University of Wisconsin, WONCA Africa, American Academy of Family Physicians, College of Family Physicians of Canada, Medical Education Partnership Initiative, Fogarty International Center, Slaight and Jericho Foundations, Canadian University Students Overseas International and Volunteer Service Overseas.

Conflict of interest: none declared.

Corresponding author: Ann Evensen, MD, University of Wisconsin School of Medicine and Public Health, Department of Family Medicine and Community Health, Madison, WI. (E-mail: ann.evensen@uwmf.wisc.edu.) 
Table 1. Comparison of Traditional DAH and GHPs

\begin{tabular}{|c|c|c|}
\hline Factor & Traditional DAH & GHPs \\
\hline Goal & $\begin{array}{l}\text { Improve health of patients and communities, decrease } \\
\text { disease burden }\end{array}$ & $\begin{array}{l}\text { Strengthen local health systems, foster personal } \\
\text { and professional growth for all partners }\end{array}$ \\
\hline Design & $\begin{array}{l}\text { Reactive, project-focused: } \\
\text { Provide HIV testing and medication } \\
\text { Malaria and tuberculosis control program } \\
\text { Local health worker education } \\
\text { Introduce technology (CT scan, mobile apps, } \\
\text { dialysis etc.) }\end{array}$ & $\begin{array}{l}\text { Proactive, system-focused: } \\
\text { Support of new medical residency program } \\
\text { Support of child nutrition program using } \\
\text { local resources (human, financial, nutrient } \\
\text { sources, distribution networks) }\end{array}$ \\
\hline Source of aid & External funding provides majority of support & $\begin{array}{l}\text { Local funding for ongoing program needs } \\
\text { supplemented by external funding for start- } \\
\text { up needs }\end{array}$ \\
\hline Focus on sustainability & $\begin{array}{l}\text { May not be high priority; intent is to match available } \\
\text { external interests/funding to potential applications } \\
\text { in a community or organization }\end{array}$ & $\begin{array}{l}\text { Typically high priority; interest exists within a } \\
\text { community to support a program after initial } \\
\text { support wanes }\end{array}$ \\
\hline Potential attributes & $\begin{array}{l}\text { Can bring significant influx of financial and expert } \\
\text { advice to a community in need. Contacts made as } \\
\text { part of traditional DAH may foster future global } \\
\text { health partnerships. }\end{array}$ & $\begin{array}{l}\text { Relationships and services developed during } \\
\text { the partnership may enable additional } \\
\text { partnership activities. Community's highest } \\
\text { needs are prioritized. May be more } \\
\text { successful in addressing root causes of } \\
\text { disease. }\end{array}$ \\
\hline Potential challenges & $\begin{array}{l}\text { Reliance on external aid reduces likelihood of } \\
\text { sustainability and may siphon local expertise and } \\
\text { funds from other needs. }\end{array}$ & $\begin{array}{l}\text { Measuring traditional DAH project deliverables } \\
\text { may be difficult or take longer. Community } \\
\text { or partner priorities may change over time, } \\
\text { decreasing chances of program sustainability. }\end{array}$ \\
\hline
\end{tabular}

Buse et $\mathrm{al}^{6}$, Rosenberg et $\mathrm{al}^{7}$, Starfield ${ }^{8}$, Olapade-Olaopa ${ }^{9}$, Melby. ${ }^{10}$

foundations have invested significantly in global health. In 2015, over \$30 billion dollars went to Development Assistance for Health (DAH) globally; $\$ 6$ billion came from the United States. ${ }^{2} \mathrm{Al}-$ though DAH has contributed to many important achievements (eg, eradication of small pox, treatment for patients living with HIV/AIDS) some of this spending may have inadvertently weakened the local health systems that it intended to help. ${ }^{3-6}$

Action through global health partnerships (GHPs) is a contrasting strategy to traditional $\mathrm{DAH}$ for accelerating progress toward global health targets. See Table 1 for a comparison of traditional DAH and GHPs. ${ }^{6-10}$ In a partnership, each partner has unique assets contributed to the benefit of all parties. Key stakeholders are able to achieve progress they could not accomplish on their own. This partnership approach is a shift away from the medical mission model-where health professionals and students from high income countries engage in short-term relief efforts in low and middle income countries-to one where all stakeholders learn and contribute. ${ }^{11,12}$

Although partnerships that coordinate shortterm relief are sometimes essential to address crises, programs that build capacity of local health systems to provide high-quality, self-sustaining medical education and health care systems create longer-lasting benefits. ${ }^{13-15}$ The aim of this article is to describe lessons learned during our multi-year collaboration consisting of bidirectional visits and complex partnerships between multiple clinicians, departments, and governments from Ethiopia and North America. The coauthors represent institutions that collaborated to design, launch, and sustain the first Family Medicine (FM) residency in Ethiopia. ${ }^{9,17-19}$ We believe that these lessons can inform the realistic development of useful GHPs, particularly in the development of FM initiatives, as well as future rigorous program evaluation.

\section{Development of Family Medicine in Ethiopia}

Ethiopia faced significant internal and external challenges leading up to and following the decision to begin FM residency training. Since the communist government (Derg) fell in 1991, the federal republic has been led by elected prime ministers with different health care workforce priorities. Domestic droughts and conflicts in neighboring Eritrea, Somalia, and Sudan/South Sudan caused suffering and death due to armed conflict, famine, and increased stress on the economy and environment from an influx of refugees. Despite these crises, health professionals across Ethiopia diligently maintained existing educational pro- 
grams and considered transforming their medical education and health care sectors. ${ }^{16}$

In 2001, University of Toronto (U of T), University of Wisconsin (UW) faculty first visited Addis Ababa University (AAU) to discuss academic collaboration in Ethiopia including residency training in FM. ${ }^{17}$ The following year, the $\mathrm{U}$ of $\mathrm{T}$ Faculty of Medicine began an academic partnership to develop a Psychiatry residency program at AAU. The Toronto Addis Ababa Academic Collaboration paired $U$ of $T$ and AAU departments to support disciplines from engineering to nursing and ultimately FM.

In North America, more than 50 years of experience with FM has created awareness and demonstrated benefits of the specialty. In contrast, Ethiopians had not experienced continuous and comprehensive care from well-trained generalists, so expectations and support for FM were initially minimal. In addition, other partnerships introduced compelling but expensive programs that competed for limited health care resources such as magnetic resonance imaging and kidney transplantation.

The benefits of a primary-care-based health care model were well established. ${ }^{8}$ Colleagues from the Ethiopian Federal Ministry of Health $(\mathrm{MoH})$ and AAU recognized FM as a means to achieving many priority goals of the Ethiopian health system. These included improving access to well-trained physicians in areas too remote to attract surgical and medical specialists, improved health indicators, and reducing costs of specialty training, physician recruitment, and avoidable medical complications.

In 2010, the $\mathrm{MoH}$ and other key stakeholders identified strengthening primary health care as a national priority. In alignment with this goal, AAU, $\mathrm{UW}$, and $\mathrm{U}$ of $\mathrm{T}$ began discussions to design and launch the first FM residency program at AAU. Support for the Ethiopian FM program development came from a number of stakeholders and funders as shown in Figure 1. ${ }^{9,18}$

The first FM residency was launched at AAU in 2013 and the first 7 Ethiopian family physicians graduated in February 2016. A detailed description of Ethiopian FM program development has been previously published. ${ }^{17}$

\section{LESSON 1: Promote Family Medicine as a Distinct Specialty}

The unique aspects of FM programs include continuity clinical training venues, community medicine/public health training, and liaisons for FM
Figure 1. Key stakeholders in the development of Family Medicine in Ethiopia.

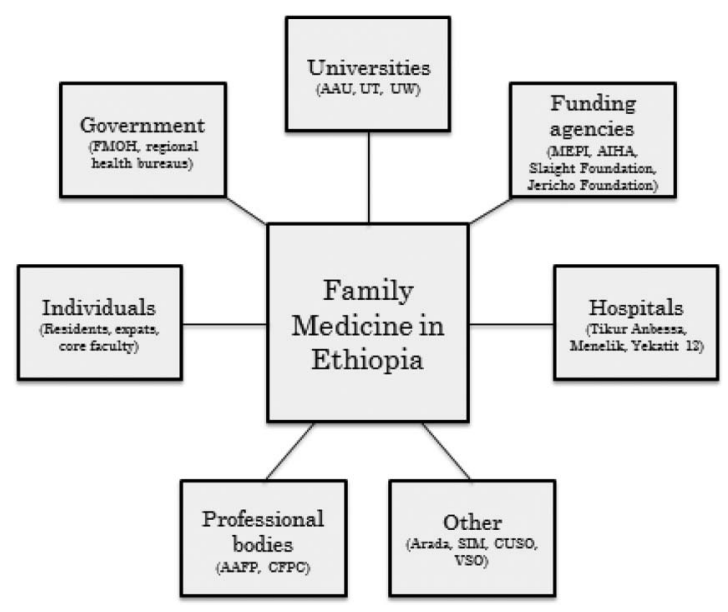

within each key specialty (internal medicine, pediatrics, surgery, psychiatry, emergency medicine and obstetrics and gynecology). Liaisons adapted the local medical education culture to meet these needs. Our partnerships enriched this dialog, providing a balance between what was possible, what was ideal, and what was realistic given resources and local political climate.

Partners agreed that the first FM program should not begin until the Ethiopian $\mathrm{MoH}$ approved FM as an official Ethiopian medical specialty. With this designation, an Ethiopian FM physician would receive a "specialist" salary that would be greater than that of a general practitioner. Strong Ethiopian leadership and recruitment of a motivated, local champion were indispensable to identify and negotiate with key stakeholders. This included a series of strategic communications, discussions, and meetings with Ethiopian health ministers.

Faculty proposed a career ladder for family physicians within the Ethiopian health system, advertised the new program, recruited a brave and capable cohort of pioneer residents, identified a cadre of Ethiopian FM-friendly specialists and consultants, and made regional connections with established African FM programs and World Organization of National Colleges, Academies and Academic Associations of General Practitioners/Family Physicians (WONCA) Africa.

Full support of FM could not be secured before starting the residency because it was not possible to know in advance the benefits and opportunities of FM. As stakeholders' understanding of the poten- 
tial of FM increased, residency leaders and supporters also increased their understanding of stakeholders needs. For example, Ethiopian Regional Health Bureaus needed physicians in rural health centers to have obstetric and general surgical skills, so increased surgical training was incorporated into the FM residency. Data were also collected to assess the needs of practicing general practitioners. ${ }^{17}$ Frequent communication and negotiation with stakeholders nurtured interest in FM, inspired additional collaborations, and recruited additional human and financial resources.

\section{LESSON 2: Avoid Gaps, Conflict, and Redundancy in Partner Priorities and Activities}

The stakeholders involved in the ongoing development of FM in Ethiopia (Figure 1) were influenced by political agendas, access to power and resources, divergent communication styles and cultural norms, differing ethical and professional expectations, and individual personality characteristics. Partners often encountered conflicting priorities such as facilitating the host's vision for the project, creating reciprocal learning and benefits for all partners, matching partnership activities to meet grant requirements, and efficiently and effectively using investments of time and expertise.

Global partners are accountable to a diverse group of funders and supervisors, often with competing expectations that may change abruptly as new leaders assume power. These changes may erode trust and trigger frustrations within GHPs if they affect priorities, funding, and definitions of "acceptable" activities.

Recognize partners' motivations and needs such as pursuit of scholarship and promotion. Institutions may seek grant funding and prestige. Partners may operate independently, resulting in competition, duplication, and gaps. This diversity of expectations can dilute and distract attention from critical needs of the communities and health systems served by a GHP.

Yet collaborator diversity provides opportunities for creative problem solving. Collaborations in global health can be compared with the variety and adaptation that naturally occurs in "ecotones," areas of transition between 2 more stable ecological communities. ${ }^{20}$ Individuals and communities in ecotones adapt and develop new and unique features not found in either border region due to the diver- sity of reciprocal, symbiotic interactions. ${ }^{20}$ This type of synergy may be beneficial in GHPs.

Recognizing competing priorities at a project's outset can improve the chances of sustaining productive, long-term partnerships. It is important to identify shared end goals, even when underlying motivations differ. Although complete harmony is often unrealistic, goals are more likely to be accomplished when all partners and their respective aims and responsibilities are clearly delineated.

\section{LESSON 3: Building Relationships Takes Time and Shared Experiences}

Respectful, trusting relationships between individuals form the nucleus around which sound international institutional collaborations form. Productive relationships benefit from time and shared experiences. Individual, informal relationships may begin years before formal collaborations; these can lead to other relationships, formal programs, and grant funding.

Relationships between Ethiopian and North American faculty began a decade before the Ethiopian FM residency was launched. These relationships were sustained through bidirectional site visits, continuing education programs, a needs assessment, a time-motion study, administrative negotiations, grant-writing, and faculty development. ${ }^{17,19}$ Some individuals had already worked together to develop the first Ethiopian Emergency Medicine residency program, ${ }^{6}$ enhance AAU's Psychiatry residency, ${ }^{21}$ establish a Gender Equity Office, ${ }^{22}$ and launch Advanced Life Support in Obstetrics in Ethiopia. ${ }^{23}$ These previous collaborations provided a foundation of trust, safety, strength and credibility for the FM residency, as well as securing additional resources for FM.

Building relationships among individuals and groups with differences in history, culture, power, access to resources, and organizations is challenging. In a critique of international public health collaborations, the anthropologist Wenzel Geissler describes the "knowing unknowing" that takes place in collaborations around issues of resources and power. ${ }^{24}$ Geissler observes that partners are aware of, but may not acknowledge, the power and resource differentials. These differentials can generate animosity that may be misattributed to personality or cultural differences.

The personalities and interests of individuals within partnerships matter. Every collaborator has 
Figure 2. Influences on relationships and decision making in global health partnerships.

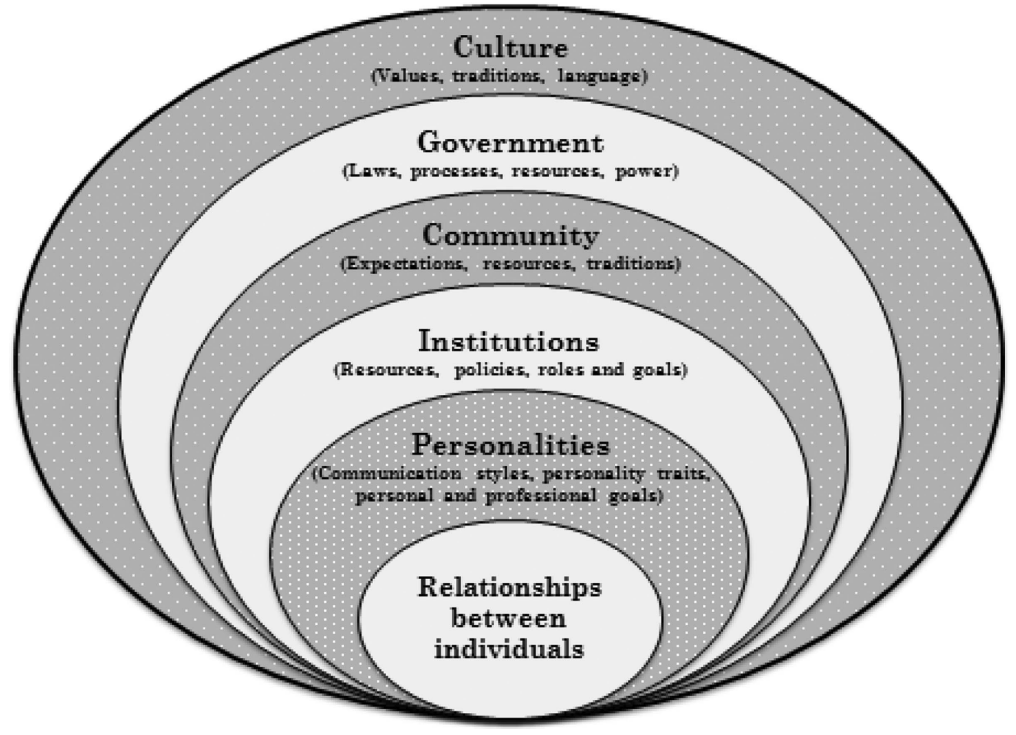

needs, resources based on past experiences, and professional, partnership and/or community responsibilities. Figure 2 illustrates the layers of influence potentially affecting individuals involved in GHPs. Strong interpersonal relationships based on affection, mutual respect, curiosity and trust can be a source of great joy and personal satisfaction. Mutual exploration of medical, academic, and local cultures is a benefit of participation in GHPs. Personal relationships can carry a program through times of reduced funding, confusion, and other uncertainties.

\section{LESSON 4: Communicate Frequently to Create Opportunities for Success}

Communication is key to sustain progress. ${ }^{25}$ Initial encounters between individuals often create longlasting impressions. However, these encounters often occur electronically, with potential for miscommunications and unreliable Internet access.

Initial encounters might include discussion of values, principles, resources, and goals to assess whether there is sufficient common ground for collaboration. Participants should be receptive to different perspectives. Cultural humility can provide opportunities to explore expectations and to handle the unexpected. ${ }^{26}$ Effective communication, in turn, may reveal previously unmet needs and opportunities.

Develop relationships with key stakeholders. For example, an Ethiopian faculty member with limited knowledge of FM was selected by the Dean of the medical school to lead the new FM residency. Fortunately, an initial meeting between the Ethiopian and North American faculty members enabled each partner to begin to understand the other and to build trust before committing to continued collaborations.

After initial meetings, the more challenging work of developing and sustaining a relationship begins. Acceptable topics and modes of communication may differ between people and cultures and lead to misunderstandings. For example, 1 partner may say "yes" out of respect when actually meaning "no" to a plan or suggestion. Verbal and nonverbal cues may be misinterpreted. Problems can be related to:

- Differing expectations regarding decision making: "Is this decision made by the partner, a person with more seniority or by individuals unknown to the consultant?"

- Misinterpretation of a reaction: "Does my partner's silence mean a lack of interest, desire to preserve the peace, or simply a lack of access to electricity or Internet?"

- Difference in timelines: "Is this deadline arbitrary or does it serve the needs of the host or funder?"

- How to provide feedback: "Am I empowered to suggest a change or correct this mistake?" 
Expect conflicts and seek opportunities to discuss disagreements, given that misunderstandings will be common. Disagreements and differences in expectations that go unrecognized or unspoken are more likely to be disruptive than those that are openly discussed. Consider trying to mutually and explicitly identify potential areas of conflict related to expectations, gaps and overlaps between hosts and consultants as well as those among multiple consultants. Goals should be pursued at a pace that is appropriate for the host and their community rather than to meet the deadlines of visitors.

\section{LESSON 5: Engage Local Leaders to Build Sustainable, Long-Lasting Programs from the Beginning of the Partnership}

Strategic planning is an iterative process of identifying goals and desired outcomes. Planning was conducted in the context of a long-term commitment recognizing the need for flexibility and responsiveness, succession planning, and stewardship of local and external resources (eg, people, time, and money). ${ }^{10}$

Each institution in our GHP was invested in a long-term relationship that transcended grants or time-dependent boundaries. We used frequent programmatic re-evaluation to identify duplicative efforts, gaps, or less fruitful endeavors. This helped facilitate honest and meaningful feedback regarding project outcomes, individual and organizational commitments, and deadlines. "Success metrics" were reviewed and redefined many times by each partner. Effective critical review required all partners to be willing listeners, receptive of recommendations to adjust or change course for the greater good of the program.

The FM program in Ethiopia used 2 tools to assist in strategic planning. WONCA guidelines for postgraduate FM training provided an external standard of competency measures (see Table 2) ${ }^{27}$. Local leaders and consultants developed a "Roadmap for Family Medicine in Ethiopia," which was adopted by the Ethiopian MoH (see Table 3$)^{28}$. FM program development was measured against these standards and changed as necessary to fill gaps.

North American partners suspended their idea of what FM "should" be in the Ethiopian context. Ethiopian partners knew that expectations for family physicians might be very different in urban versus rural areas. Ethiopian training sites and com-
Table 2. WONCA Global Standards for Postgraduate

\section{Family Medicine Education}

Comprehensive document addressing the structure, process and outcome of postgraduate medical education and training:

1) Mission and Outcomes

2) Training Process (structured curriculum that is comprehensive, community-based, continuity of care, care for undifferentiated problems and psychosocial needs; service vs learning)

3) Assessment of trainees (including feedback)

4) Trainees (admission policies, working conditions, support)

5) Staffing (appointments, obligations)

6) Training settings and educational resources (inpatient and outpatient, information technology, team-based care)

7) Evaluation of training process (trainee evaluation of program, using trainee competence as measure, involvement of stakeholders)

8) Governance and administration (leadership, funding, administration)

9) Continuous renewal (quality improvement of program based on needs of communities, institutions and trainees

WONCA Working Party on Education. ${ }^{27}$

munities could draw on FM's flexibility and adaptiveness to design programs to train clinicians who could best meet the needs of different communities.

Sustainability was achieved through support by local leaders and by combining external and local

Table 3. Highlights from the Ethiopian Federal Ministry of Health Roadmap for Family Medicine

The Roadmap was created by using landmark publications regarding the value of family medicine and fundamental components of training programs combined with the authors' experience of establishing the first Ethiopian family medicine program.

Summarizes the value of family medicine in general, in the African context, and in Ethiopia.

Recognizes that incorporating family medicine will offer improvements and efficiencies in service and improve access to health care.

Suggests steps for a well-planned and careful implementation of FM in Ethiopia:

1) Define clear but flexible role for FM in Ethiopian health care system based on both countrywide and local needs and resources. Align role of FM with other primary health care providers.

2) Recruit trainees and establish training programs. Collaborate with international partners to provide faculty until sufficient Ethiopian family physicians have been trained.

3) Introduce family physicians via a pilot program where 2 to 4 physicians lead a primary health care team in a local health care system.

WONCA. ${ }^{28}$ 
funding. Local funding was used for Ethiopian staff and resident salaries, purchasing supplies, and other local costs. External funding was used primarily for start-up costs to avoid creating a program that would require external funding by international partners.

As the value of FM grew, the GHP expected program and salary costs would be provided by the Ethiopian medical education and health care systems. AAU now funds the FM program and the $\mathrm{MoH}$ has agreed to expand FM training to Jimma University and University of Gondar, 2 well-established medical schools located in cities several hours away from the capital.

Effective stewardship of human resources by local and international partners was critical for longterm success. Orientation and hosting of North American partners requires local partners to take time away from family, clinical, and administrative responsibilities. Well-intended but short visits by multiple visiting faculty, all whom require orientation, are more burdensome than a single faculty member visiting for an extended time. To ensure a balance of benefits and effort, hosts worked to select visitors who could provide high-yield contributions and avoid unnecessarily taxing local resources.

Although founding partners, relationships, and individuals must be celebrated, there was also a need for succession planning. The introduction of new and energetic colleagues may allow a GHP to undertake the next phase in its growth. The AAU program has new leadership and faculty, including Ethiopian FM graduates and expatriate family physicians committed to remain for several years. North American partners recruited new faculty as well. The external partners plan to decrease their involvement over time as the Ethiopian FM graduates increase in number and are able to assume leadership roles. The ultimate goal is that Ethiopian Family Medicine will thrive and grow without the need for external support.

\section{Conclusions}

Relationships between family physicians, health professional colleagues, patients and communities form the heart of FM in Ethiopia and North America alike. Relationships between individuals and institutions are also the basis of strong global health collaborations. Shared vision, goals, values and strategies are more likely to result in mutually beneficial GHPs and positive outcomes. Frequent, consistent communications, bidirectional visits and complementary (rather than competing) activities enhance development of site-appropriate methods and models. Partnerships, processes and goals that are sensitive and responsive to local priorities, context and culture are more likely to thrive and to be sustained.

Despite the most carefully laid plans, the uncertainties, ambiguities and serendipity of GHPs often result in an unpredictable series of steps and accomplishments. Expect the unexpected and patiently keep the end goals in mind. Positive relationships, persistence, and recognizing and responding to opportunities when they arise are the keys to success.

GHPs inevitably provide opportunities for the meeting of people, ideas, and cultures. All partners are bound to grow and change as they give, receive, and enrich their mutual understanding. Despite the challenges, GHPs provide rich rewards as they unite the human family around shared goals of promoting health for all.

The authors gratefully acknowledge Mindy Smith, MD for review of this manuscript and to the many partners supporting the Addis Ababa University Family Medicine Program: University of Toronto, University of Wisconsin, WONCA Africa, American Academy of Family Physicians, College of Family Physicians of Canada, Medical Education Partnership Initiative, Fogarty International Center, Slaight and Jericho Foundations, Canadian University Students Overseas International, and Volunteer Service Overseas.

To see this article online, please go to: http://jabfm.org/content/ 30/5/670.full.

\section{References}

1. United Nations. The 2030 agenda for sustainable development and its 17 sustainable development goals. Available from: http://www.un.org/sustain abledevelopment/sustainable-development-goals/ Accessed on April 7, 2017.

2. Institute for Health Metrics and Evaluation. Data visualizations. Available from: http://www.health data.org/results/data-visualizations. Accessed on April 7, 2017.

3. Syed SB, Dadwal V, Rutter P, et al. Developeddeveloping country partnerships: Benefits to developed countries? Global Health 2012;8:17.

4. Crisp N. Turning the world upside down: The search for global health in the 21 st century. 1st ed. London, UK: CRC Press, 2010.

5. Spicer N, Aleshkina J, Biesma R, et al. National and subnational HIV/AIDS coordination: Are global 
health initiatives closing the gap between intent and practice? Globalization and Health 2010;6:3.

6. Busse H, Azazh A, Teklu S, et al. Creating change through collaboration: A twinning partnership to strengthen emergency medicine at Addis Ababa University/Tikur Anbessa Specialized Hospital. Academic Emergency Medicine 2013;20:1310-8.

7. Rosenberg ML, Hayes ES: McIntyre MH, et al. Real collaboration: What it takes for global health to succeed. Berkeley, CA: University of California Press, 2010.

8. Starfield B. Global Health, equity, and primary care. Am Board Fam Med 2007;20:511-3.

9. Olapade-Olaopa EO, Baird S, Kiguli-Malwadde E, et al. Growing partnerships: Leveraging the power of collaboration through the Medical Education Partnership Initiative. Acad Med 2014;89(8 Suppl): S19-23.

10. Melby M, Loh L, Evert J, et al. Beyond medical "missions" to impact-driven short-term experiences in global health (STEGHs): Ethical principles to optimize community benefit and learner experience. Acad Med 2016;91:633-638.

11. Boydell L, Rugkasa J, Hoggett P, et al. Partnerships: the benefits. 1st ed. Dublin, Ireland: Institute of Public Health in Ireland, 2007. Available from: http://www.publichealth.ie/sites/default/files/ documents/files/IPHbenefits.pdf. Accessed on April 7, 2017.

12. United Nations Global Compact: Catalyzing transformational partnerships between the United Nations and business. New York, NY: UN Global Compact Office, 2011. Available from: https://www. unglobalcompact.org/docs/issues_doc/un_business_ partnerships/Catalyzing_Transformational_Partnerships. pdf. Accessed on April 7, 2017.

13. Farmer P, Rhatigan J. Embracing medical education's global mission. Acad Med 2016;91:1592-4.

14. World Health Organization. Everybody's business: Strengthening health systems to improve health outcomes: WHO's framework for action. Geneva, Switzerland: World Health Organization, 2007. Available from: www.who.int/healthsystems/ strategy/everybodys_business.pdf. Accessed on April 7, 2017.

15. International Federation of Pharmaceutical Manufacturers Association. Working toward transformative health partnerships in low- and middle-income countries. 2012.

16. Federal Democratic Republic of Ethiopia, Ministry of Health. Health sector development program IV, 2010/11-2014/15. October 2010. Available from: http://phe-ethiopia.org/admin/uploads/attachment-
$721-\mathrm{HSDP} \% 20 \mathrm{IV} \% 20 \mathrm{Final} \% 20 \mathrm{Draft} \% 2011$ Octoberr\%202010.pdf. Accessed on April 7, 2017.

17. Philpott J, Cornelson B, Derbew M, et al. The dawn of family medicine in Ethiopia. Fam Med 2014;46: 685-90.

18. Toronto Addis Ababa Academic Collaboration. Available from: http://taaac.com. Accessed on April 7, 2017.

19. Philpott J, Derbew M. Use of a CME workshop to introduce and promote the specialty of Family Medicine in Ethiopia. Afr J Prim Health Care Fam Med 2010;2(1).

20. Seidman P. Vitality at the edges: Ecotones and boundaries in ecological and social systems. World Future Review 2009;1:31-47.

21. Alem A, Pain C, Araya M, et al. Co-creating a psychiatric resident program with Ethiopians, for Ethiopians, in Ethiopia: The Toronto Addis Ababa Psychiatry Project (TAAPP). Acad Psychiatry 2010;34: 424-32.

22. Kvach E, Desalegn D, Conniff J, et al. Promoting gender equity at the College of Health Sciences Addis Ababa University, Ethiopia. Ethiopia Med J 2015;(Supp. 2):9-16.

23. Evensen A, Panzer J, Jones S, et al. Improving confidence in obstetric skills with Basic and Advanced Life Support in Obstetrics ${ }^{\circledR}$ training in Ethiopia: A pre/post study. J Woman's Reprod Health 2015;1: 24-33.

24. Geissler PW. Public secrets in public health: Knowing not to know while making scientific knowledge. Am Ethnologist 2013;40:13-34.

25. Kraeker C. "We learn from them, they learn from us": Global health experiences and host perceptions of visiting health care professionals. Acad Med 2013; 88(4).

26. Tervalon M, Murray-Garcia J. Cultural humility versus cultural competence: A critical distinction in defining physician training outcomes in multicultural education. J Health Care Poor Underserved 1998;9:117-25.

27. WONCA Working Party on Education. WONCA global standards for postgraduate family medicine education. 2013. Available from: http://www.global familydoctor.com/site/DefaultSite/filesystem/ documents/Groups/Education/WONCA\%20ME \%20stds_edit\%20for\%20web_250714.pdf. Accessed on April 7, 2017.

28. WONCA. Family Medicine Ethiopia Highlights, February 2015. Available from: http://www.global familydoctor.com/News/FamilyMedicineEthiopia Highlights.aspx. Accessed on April 7, 2017. 